\author{
Se Hwan Kwon \\ Joo Hyeong Oh
}

\section{A large vertebral artery pseudoaneurysm due to percutaneous internal jugular vein cannulation}

Received: 28 July 2014

Accepted: 6 August 2014

Published online: 15 August 2014

(C) Springer-Verlag Berlin Heidelberg and ESICM 2014

\section{S. H. Kwon $(\bowtie) \cdot J$. H. Oh}

Department of Radiology, Kyung Hee University Medical Center, College of Medicine, Kyung Hee University, 23, Kyungheedae-ro, Dongdaemun-gu, Seoul 130-872, Republic of Korea e-mail: kwon98@khu.ac.kr

Tel.: 82-2-958-8622

A 60-year-old man presented with an exacerbation of pulmonary edema, pneumonia, and lethargy. The patient had undergone attempted hemodialysis catheter placement in the right internal jugular vein (IJV) without ultrasonographic guidance. During the procedure, the patient developed increased stupor with a pulse of 30 beats per min and a blood pressure of 50/30 $\mathrm{mmHg}$. Emergency cardiopulmonary resuscitation was performed. Chest x-ray imaging showed a bulging contour at the right paratracheal area with supra-mediastinal widening (Fig. 1a). On chest computed tomography (CT), a large pseudoaneurysm was identified at the right-side supra-mediastinum with surrounding hematoma. The pseudoaneurysm originated from the proximal right vertebral artery (VA) (Fig. 1b). Selective right subclavian angiography confirmed a large pseudoaneurysm of the proximal portion of the right VA. Three metal coils were deployed to occlude the proximal right VA. Then, a $10 \times 25 \mathrm{~mm}$ balloon-expandable stent graft was deployed into the right subclavian artery (SCA) across the VA origin. After the procedure, angiography revealed no filling of the pseudoaneurysm and patency of the right SCA.

Most catheterization-related VA injuries occur during IJV catheterization. Prevention is the best treatment for all iatrogenic arterial injuries. Ultrasound-guided IJV catheterization should be performed whenever available.
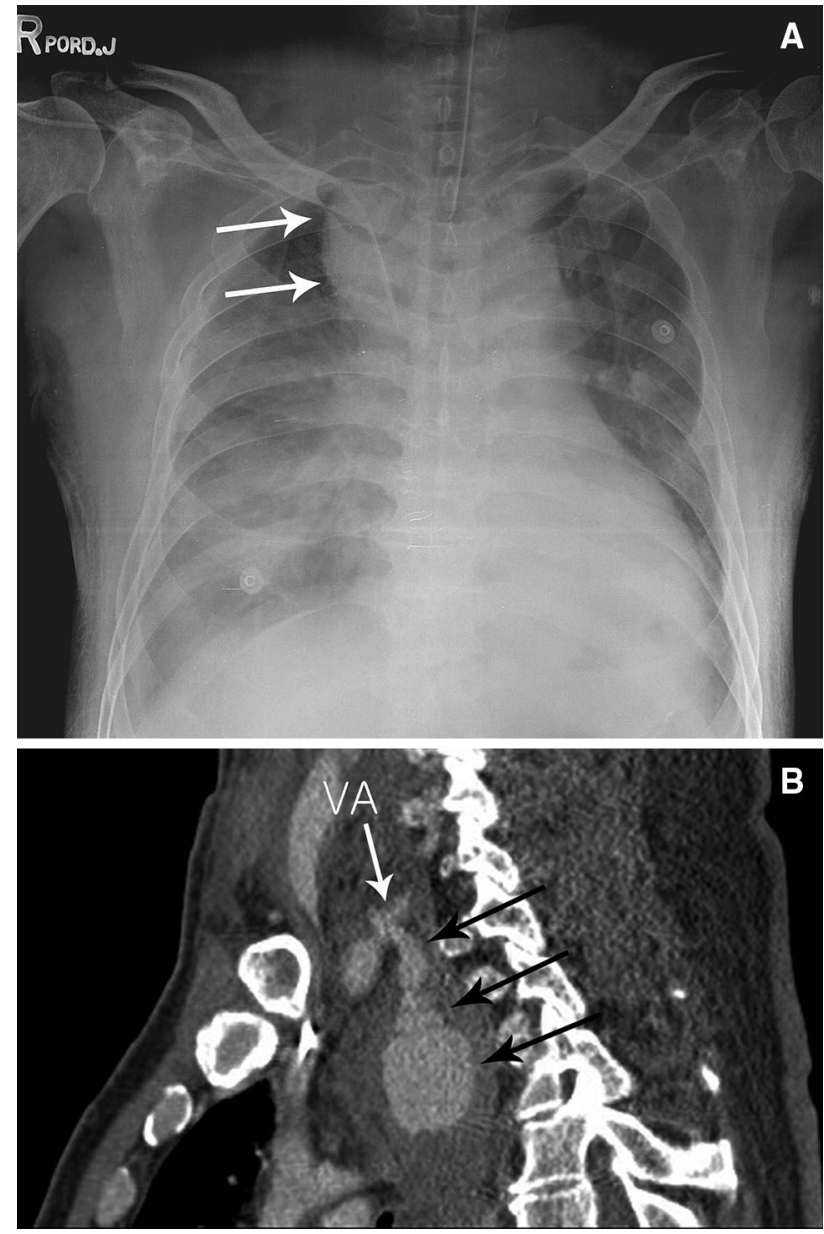

Fig. 1 a Plain chest $x$-ray showing the bulging contour of the right paratracheal area (arrows) and supra-mediastinal widening. b Oblique sagittal multiplanar reformatted CT image showing a large pseudoaneurysm (black arrows) at the right-side supra-mediastinum with surrounding hematoma and the origin of the pseudoaneurysm at the proximal right vertebral artery (VA, white arrow)

Conflicts of interest All authors have nothing to disclose. 